University of Nebraska - Lincoln

DigitalCommons@University of Nebraska - Lincoln

\title{
Fertilizing capacity and morphology of fowl and turkey spermatozoa in hypotonic extender
}

\author{
M. R. Bakst \\ United States Department of Agriculture
}

Follow this and additional works at: https://digitalcommons.unl.edu/usdaarsfacpub

Part of the Agricultural Science Commons

Bakst, M. R., "Fertilizing capacity and morphology of fowl and turkey spermatozoa in hypotonic extender" (1980). Publications from USDA-ARS / UNL Faculty. 632.

https://digitalcommons.unl.edu/usdaarsfacpub/632

This Article is brought to you for free and open access by the U.S. Department of Agriculture: Agricultural Research Service, Lincoln, Nebraska at DigitalCommons@University of Nebraska - Lincoln. It has been accepted for inclusion in Publications from USDA-ARS / UNL Faculty by an authorized administrator of DigitalCommons@University of Nebraska - Lincoln. 


\title{
Fertilizing capacity and morphology of fowl and turkey spermatozoa in hypotonic extender
}

\author{
M. R. Bakst \\ U.S. Department of Agriculture, Science and Education Administration, Agricultural Research, \\ Avian Physiology Laboratory, Beltsville, Maryland 20705, U.S.A.
}

\begin{abstract}
Summary. The effects of graduated increments of hypotonic Beltsville Poultry Semen Extender (BPSE) on fowl and turkey sperm morphology, motility, percentage dead and fertility were examined. The morphology of fowl and turkey spermatozoa was adversely affected by decreases in BPSE osmolarity; a reduction of osmolarity of BPSE to below 200 and 140 mosmol adversely affected the fertility of fowl and turkey spermatozoa, respectively. The addition of Ficoll to the extender allowed direct observation of the effects of hypotonic BPSE on the sperm plasmalemma. Plasmalemmal swelling, due to the uptake of water, was noted initially at the midpiece. Such plasmalemmal swelling may predispose spermatozoa to further osmotic-induced damage.
\end{abstract}

\section{Introduction}

The damage caused to fowl and turkey spermatozoa by the addition of diluents and cryoprotective agents and freezing and thawing have been documented (Polge, 1951; Harris, Thurston \& Cundall, 1973; Westfall \& Harris, 1975; Sexton, 1976; Oderkirk \& Buckland, 1976; Marquez \& Ogasawara, 1977; Bakst \& Sexton, 1979). Although gross anomalies such as bent spermatozoa (also called crook-neck (Saeki, 1960) and neck-bending (Yamane, Tsukunaga \& Takahashi, 1966; Van Wambeke, 1977) spermatozoa), tail coiling, and mitochondrial swelling are discernible with bright-field light microscopy, plasmalemma integrity is difficult if not impossible to discern. Drevius \& Ericksson (1966) devised a method whereby plasmalemma integrity could be determined by phase-contrast microscopy: bovine spermatozoa were placed in a hypotonic medium with a refractive index greater than that of the sperm cytosol and areas of plasmalemma distension resulting from the osmotic uptake of water were visualized. Such areas appeared as bright spots or halos and were only seen with spermatozoa that had an intact plasmalemma. The degree of plasmalemma distension, i.e. water uptake, by the spermatozoa was directly related to the tonicity of the medium, demonstrating that bull spermatozoa behave as osmometers (Drevius, 1972). Hypotonic media also induced partial or complete coiling of the axoneme, depending on the tonicity of the medium.

The similarity between the morphological alterations of bull spermatozoa in hypotonic media (Drevius \& Ericksson, 1966) and fowl and turkey spermatozoa after the addition of cryoprotective agents and freezing and thawing (Bakst \& Sexton, 1979) prompted the present study to (1) examine the effects of hypotonic semen extender on morphological and physiological characteristics of fowl and turkey spermatozoa, and (2) devise a method for the rapid assessment of sperm plasmalemmal integrity. 


\section{Materials and Methods}

Semen collection and preparation were identical for the fowl and turkey. Semen was collected by abdominal massage from 5 or more males of known fertility, and was pooled and diluted with an equal volume of Beltsville Poultry Semen Extender (BPSE) (see Sexton, 1977, for composition). Equal aliquots of BPSE were made hypotonic $(80,60,40,20,0 \%$ BPSE), in duplicate, with distilled water. To increase the refractive index of the diluted BPSE, Ficoll (Sigma, Type 400), which is a nonionic, high molecular weight (about 400000$)$ sucrose polymer, was added $(20 \%$ $\mathrm{w} / \mathrm{v}$ ) to one aliquot of each dilution. After the osmolarity of each aliquot was measured with an automatic osmometer, one part diluted semen was added to 10 parts of each of the BPSE aliquots (with and without $20 \%$ Ficoll) and equilibrated for $15 \mathrm{~min}$. Spermatozoa in all treatments were then evaluated for morphology, motility and percentage dead. Progressive sperm motility was evaluated with a phase-contrast microscope at a magnification of $\times 400$ by examining several areas of a slide and estimating the relative number of spermatozoa moving vigorously in a forward direction. The scoring was 5 for $>90 \%$ spermatozoa moving forward: 4 for $70-89 \% ; 3$ for $40-69 \% ; 2$ for $20-39 \% ; 1$ for $<20 \%$; and 0 for no progressive motility. The percentage of dead spermatozoa was determined with a live/dead stain. Two drops of semen were added to $1 \mathrm{ml}$ of stain (5\% nigrosin and $1 \%$ eosin). Smears were made on clean slides after $2 \mathrm{~min}$, air dried and examined within $24 \mathrm{~h}$. Any spermatozoon with a pinkish colouration was considered as dead at the time of staining. Sperm morphology was assessed under oil immersion with a phase-contrast microscope.

The fertilizing capacity of fowl and turkey spermatozoa in 100\% BPSE and in hypotonic BPSE without Ficoll was tested by inseminating $6-8$ fowl and turkey hens per treatment once a week for 4 consecutive weeks with about $150 \times 10^{6}$ (fowl) and $250 \times 10^{6}$ (turkey) spermatozoa. Eggs were collected daily, held in a cool room and incubated weekly in batches. On Day 7 of incubation the eggs were candled for viable embryos; percentage fertility was expressed as an average value for each hen for the 4 -week period. Fertility was also ascertained in turkeys at 2,3 , and 4 weeks after the last of the weekly inseminations. Percentage fertility was expressed as an average value for each hen for extra week. In addition, 7 fowl were inseminated with $100 \%$ BPSE plus $20 \%$ Ficoll to determine whether Ficoll affected sperm fertility. Data were compared by analysis of variance according to the General Linear Models Procedure (Barr, Goodnight, Sell \& Helwig, 1976).

\section{Results}

\section{Morphology}

The morphology of undiluted fowl and turkey spermatozoa was identical when viewed with phase-contrast microscopy. The spermatozoa were filiform in shape and had a thick head region which tapered slightly at the midpiece and again at the annulus. The damage to the fowl and turkey spermatozoa was very similar. In the following descriptions the illustrations of a particular anomaly are given regardless of the species.

Fowl. The percentages of abnormal spermatozoa in $100 \%$ and $80 \%$ BPSE with and without Ficoll were similar (Table 1). Most abnormal spermatozoa in $100 \%$ BPSE with and without Ficoll were bent and a few were knotted. Occasionally the acrosome appeared as a small bulbous sphere at the anterior tip of the head region (Pl. 1, Fig. 1). Bent spermatozoa were characterized by an acute flexion of the tail which brought it adjacent and parallel to the head. The site of tail flexion was not only limited to the midpiece but was seen at the nucleus around the neck region and also along the proximal portion of the principal piece. Spermatozoa bent around the proximal portion of the principal piece formed a loop that enclosed a halo in Ficoll-containing solutions (Pl. 1, Figs 3 and 5; Pl. 2, Fig. 8). The presence of a halo within the loop indicated that 
the plasmalemma at this region was greatly distended and that the bend of the axoneme was within the distended plasmalemma. Bends at the midpiece were generally more acute than those at the principal piece (Pl. 2, Figs 8 and 9) but halos could still be seen at the flexure. Knotted spermatozoa were relatively rare; the tail being bent through $360^{\circ}$ to form a ' $k$ not' at the axis of the rotation (Pl. 2, Fig. 10).

Table 1. Effects of hypotonic BPSE and Ficoll on fowl spermatozoa

\begin{tabular}{|c|c|c|c|c|c|}
\hline \multicolumn{2}{|c|}{ BPSE } & \multirow{2}{*}{$\begin{array}{l}\text { Tonicity } \\
\text { (mosmol) }\end{array}$} & \multicolumn{3}{|c|}{ Spermatozoa } \\
\hline Conc. (\%) & Ficoll & & Motility & $\%$ Dead & $\%$ Abnormal \\
\hline 100 & - & $324.2 \pm 10.4$ & $4.0 \pm 0.6^{\mathrm{a}}$ & $4.8 \pm 1.6^{b}$ & $12 \cdot 4 \pm 5 \cdot 6^{b}$ \\
\hline 100 & + & $422.0 \pm 10.9$ & $2.8 \pm 0.2^{\mathrm{ab}}$ & $5 \cdot 1 \pm 2 \cdot 0^{\mathrm{b}}$ & $9.8 \pm 4.6^{\mathrm{b}}$ \\
\hline 80 & - & $262.6 \pm 8.5$ & $4.2 \pm 0.4^{\mathrm{a}}$ & $4.3 \pm 0.7^{6}$ & $19 \cdot 2 \pm 2 \cdot 1^{\mathrm{b}}$ \\
\hline 80 & + & $347.0 \pm 8.1$ & $2.6 \pm 0.4^{\mathrm{abc}}$ & $3.1 \pm 0.7^{b}$ & $23.4 \pm 9.9^{b}$ \\
\hline 60 & - & $199.2 \pm 6.3$ & $4.0 \pm 0.5^{\mathrm{a}}$ & $6.3 \pm 1.8^{\mathrm{b}}$ & $88.6 \pm 5.4^{b}$ \\
\hline 60 & + & $273.0 \pm 6.8$ & $2.8 \pm 0.6^{\mathrm{ab}}$ & $5.6 \pm 1.9^{b}$ & $86 \cdot 2 \pm 5.4^{\mathrm{a}}$ \\
\hline 40 & - & $136.0 \pm 4.2$ & $2.2 \pm 0.4^{b c}$ & $7 \cdot 6 \pm 2 \cdot 1^{\mathrm{b}}$ & $96 \cdot 2 \pm 3 \cdot 3^{a}$ \\
\hline 40 & + & $204.0 \pm 6 \cdot 2$ & $2.6 \pm 0.2^{\mathrm{abc}}$ & $11.9 \pm 5.7^{\mathrm{b}}$ & $100 \cdot 0 \pm 0^{\mathrm{a}}$ \\
\hline 20 & - & $71.0 \pm 2.3$ & $1.0 \pm 0^{\text {cd }}$ & $8.4 \pm 1.4^{b}$ & $100 \cdot 0 \pm 0^{a}$ \\
\hline 20 & + & $125 \cdot 0 \pm 2 \cdot 3$ & $1 \cdot 0 \pm 0^{\text {cd }}$ & $6.7 \pm 1.7^{b}$ & $100 \cdot 0 \pm 0^{\mathrm{a}}$ \\
\hline 0 & - & $0.8 \pm 0.4$ & $0^{\mathrm{d}}$ & $63 \cdot 6 \pm 21 \cdot 5^{\mathrm{a}}$ & $100 \cdot 0 \pm 0^{\mathrm{a}}$ \\
\hline 0 & + & $51.2 \pm 1.8$ & $0^{d}$ & $24.1 \pm 15 \cdot 3^{\mathrm{b}}$ & $100 \cdot 0 \pm 0^{\mathrm{a}}$ \\
\hline
\end{tabular}

Values are mean \pm s.e.m. of 5 replicates.

Values with different superscript letters are significantly different $(P<0.05)$.

In the $80 \%$ BPSE with Ficoll, distension of the plasmalemma at the midpiece was indicated by a faint halo in otherwise morphologically normal spermatozoa (Pl. 1, Fig. 3). The amount of damage to spermatozoa in the other concentrations of BPSE, with and without Ficoll, was similar although the type of damage varied. Bent spermatozoa predominated in 60 and $40 \%$ BPSE alone and with Ficoll, halos indicated plasmalemma distension at the midpiece and the distal tip of the tail (Pl. 1, Fig. 4). The tail anomaly, referred to as a 'tail vesicle', was the result of a partial coiling of the axoneme within the dilated plasmalemma and was less conspicuous in BPSE without Ficoll (compare Pl. 1, Fig. 4 to Pl. 2, Fig. 8). Spermatozoa with tail vesicles were motile, but more sluggish than normal spermatozoa, and generally had a halo at the midpiece. Most bent spermatozoa had a halo at the point of flexure which in the more hypotonic solutions (40\% BPSE and lower) occasionally extended anteriorly and engulfed the whole head region, indicating extensive plasmalemma distension (P1. 1, Fig. 6; P1. 1, Fig. 11). Swollen and dislodged mitochondria were seen at the midpiece in 0\% BPSE with (Pl. 2, Fig. 11) and without (Pl. 1, Fig. 2) Ficoll.

All motile spermatozoa in the $20 \%$ BPSE with and without Ficoll were morphologically abnormal. Anomalies such as dislodged mitochondria, bulbous acrosomes and bent spermatozoa were observed singly or together. In 20 and $0 \%$ BPSE plus Ficoll, halos were rarely viewed overlying the anterior and mid portions of the head, but remained associated with the midpiece and coiled tail (Pl. 1, Fig. 6; Pl. 2, Figs 11 and 12). However, the halos clearly migrated back and forth along the full length of the head and coiled tail of some immotile spermatozoa. In $0 \%$ BPSE, the sperm tail was coiled up to the midpiece and some acrosomes were observed separated from the nucleus (P1. 1, Fig. 2). In 0\% BPSE, some spermatozoa exhibited jerky movements while the coiled tail slowly undulated within the distended plasmalemma. Immotile, straight spermatozoa in hypotonic BPSE without Ficoll (Pl. 1, Fig. 2) and immotile, straight spermatozoa lacking a halo in BPSE with Ficoll (Pl. 2, Fig. 11) were assumed to have a damaged plasmalemma and were considered dead.

Turkey. Turkey spermatozoa showed nearly identical anomalies to those of fowl spermatozoa even though they were less tolerant to mild hypotonic treatments (Table 2). Most of 
the spermatozoa appeared normal in 100\% BPSE without Ficoll (Pl. 2. Fig. 7) although bent and knotted spermatozoa were present (Pl. 2. Fig. 10). A faint halo was seen at the midpiece in some spermatozoa of normal appearance in 100\% BPSE with Ficoll. In $80 \%$ BPSE only bent spermatozoa were motile. Morphologically normal spermatozoa adhered to the coverslip or slide by their anterior head region and slowly oscillated. The numbers of abnormal spermatozoa were significantly less in the presence of Ficoll in the $80 \%$ BPSE and again only the bent spermatozoa were motile and had halos at the site of tail flexion and at the tail vesicles. Swollen and displaced mitochondria were also noted in some cells (Pl. 2, Fig. 8). Those morphologically normal spermatozoa that adhered to the glass surfaces and slowly oscillated also had halos at the midpiece and formed tail vesicles. Immotile but morphologically normal spermatozoa that did not have a halo in the presence of Ficoll were presumed dead.

\section{PLATE 1}

Fowl spermatozoa in hypotonic BPSE with and without Ficoll. All micrographs were taken with a phase-contrast microscope and the magnification is $\times 560$.

Fig. 1. Bulbous acrosomes can be seen (arrows). 20\% BPSE without Ficoll.

Fig. 2. In 0\% BPSE without Ficoll sperm tails are coiled. Midpiece disruption is evident in all spermatozoa (arrowheads) and one spermatozoon has a broken acrosome (arrow). The straight spermatozoon is dead.

Fig. 3. Plasmalemma distension manifested as relatively small, faint halos is observed for some spermatozoa at the midpiece (thin arrows) and at the site of tail flexion at the midpiece (arrowhead). Another bent spermatozoon is looped at the principal piece (thick arrow). $80 \%$ BPSE with Ficoll.

Fig. 4. Spermatozoa in 60\% BPSE with Ficoll form tail vesicles (arrows) and are bent. The bent spermatozoon has a coiled tail and distended plasmalemma around the head region.

Fig. 5. In 20\% BPSE with Ficoll spermatozoa with an intact plasmalemma form halos. A tail vesicle (arrow) and plasmalemma distension at the midpiece (arrowhead) are evident.

Fig. 6. In 0\% BPSE plus Ficoll halo formation is extensive and includes portions of the head region. Mitochondrial swelling is not as evident in fowl as in turkey spermatozoa.

\section{PLATE 2}

Turkey spermatozoa in hypotonic BPSE with and without Ficoll. All micrographs were taken with a phase-contrast microscope, and the magnification is $\times 560$ except for Fig. 12 which is at $\times 160$.

Fig. 7. In 100\% BPSE without Ficoll sperm heads and midpieces appear morphologically normal.

Fig. 8. Spermatozoa in $60 \%$ BPSE without Ficoll exhibit some mitochondrial displacement (arrowhead) and tail vesicles. Bent spermatozoa with the flexure at the midpiece (thin arrows) and with a looped principal piece (thick arrow) are observed.

Fig. 9. Spermatozoa in 40\% BPSE without Ficoll exhibit considerable tail coiling and midpiece disruption. The dense granules enclosed by the coiled tails are displaced mitochondria.

Fig. 10. Two knotted spermatozoa (arrowheads) and a spermatozoon with a disrupted midpiece (thick arrow) are evident. $100 \%$ BPSE with Ficoll.

Fig. 11. All live spermatozoa in 20\% BPSE with Ficoll exhibit a halo around the midpiece or head region. Swollen mitochondria give the midpiece an irregular appearance. Some acrosomes are separated slightly from the nucleus (arrow). Dead spermatozoa lack a halo (arrowhead).

Fig. 12. A survey micrograph of spermatozoa in 0\% BPSE with Ficoll illustrates the extent of halo formation. Straight spermatozoa without halos are presumed dead (arrow). 
PLATE 1
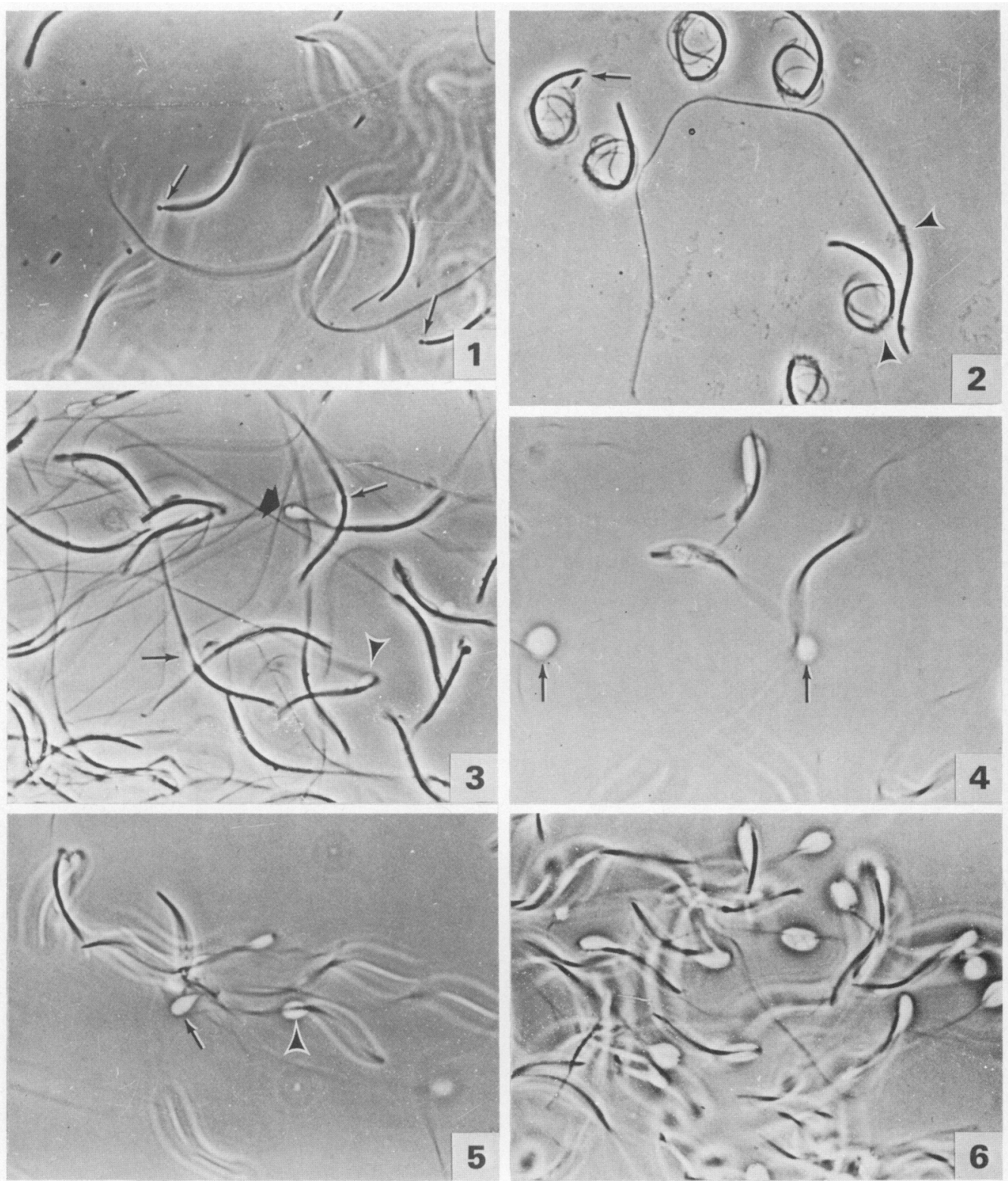

(Facing p. 124) 
PLATE 2
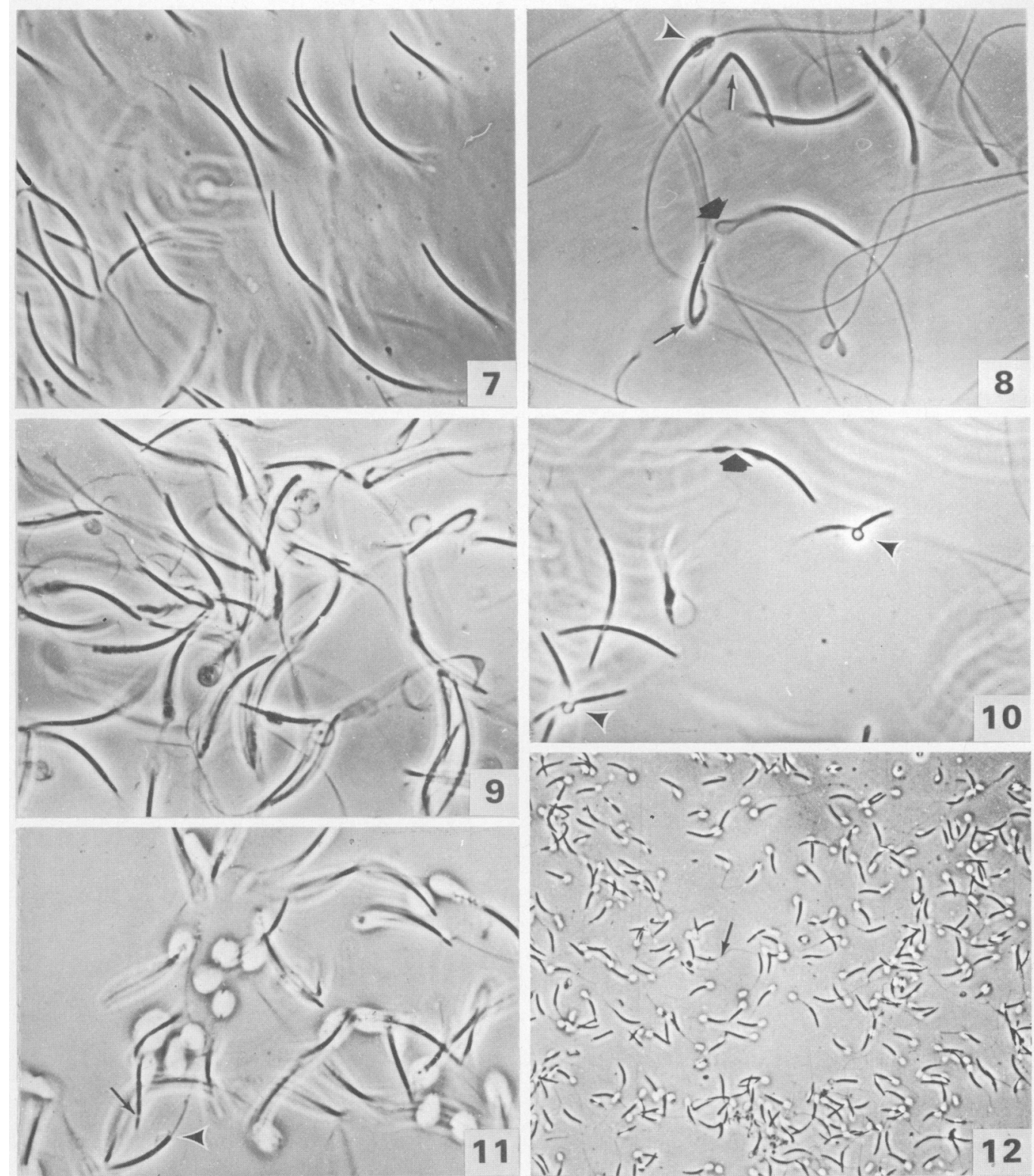

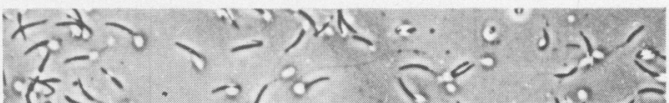

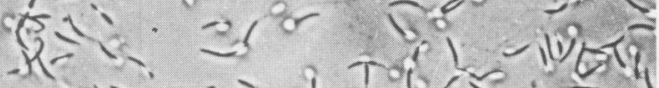
$-y=7$ ? 1 a $16=5$

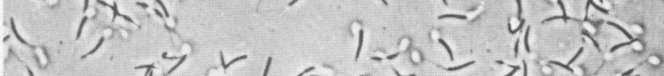
(1)

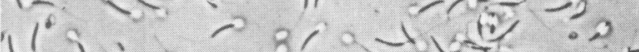

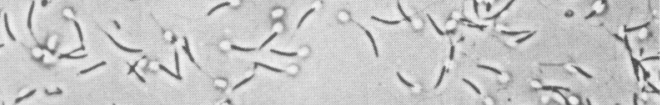

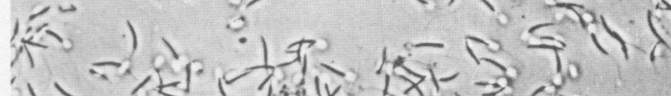

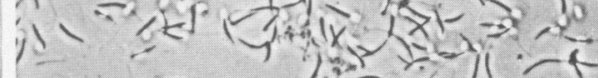


Table 2. Effects of hypotonic BPSE and Ficoll on turkey spermatozoa

\begin{tabular}{cccccc}
\hline \multicolumn{2}{c}{ BPSE } & \multicolumn{3}{c}{ Spermatozoa } \\
\cline { 4 - 6 } Conc. (\%) & Ficoll & $\begin{array}{c}\text { Tonicity } \\
\text { (mosmol) }\end{array}$ & Motility & $\%$ Dead & $\%$ Abnormal \\
\hline 100 & - & $329 \cdot 5 \pm 11 \cdot 6$ & $3 \cdot 75 \pm 0 \cdot 7^{\mathrm{ab}}$ & $2 \cdot 8 \pm 1 \cdot 1^{\mathrm{b}}$ & $17 \cdot 5 \pm 5 \cdot 7^{\mathrm{c}}$ \\
100 & + & $416 \cdot 5 \pm 14 \cdot 9$ & $2 \cdot 75 \pm 0 \cdot 5^{\mathrm{bc}}$ & $1 \cdot 9 \pm 0 \cdot 4^{\mathrm{b}}$ & $26 \cdot 8 \pm 15 \cdot 9^{\mathrm{c}}$ \\
80 & - & $266 \cdot 5 \pm 10 \cdot 1$ & $2 \cdot 75 \pm 0 \cdot 5^{\mathrm{ab}}$ & $6 \cdot 8 \pm 2 \cdot 6^{\mathrm{b}}$ & $55 \cdot 0 \pm 9 \cdot 1^{\mathrm{b}}$ \\
80 & + & $343 \cdot 75 \pm 11 \cdot 9$ & $2 \cdot 5 \pm 0 \cdot 3^{\mathrm{bc}}$ & $2 \cdot 1 \pm 0 \cdot 8^{\mathrm{b}}$ & $31 \cdot 4 \pm 6 \cdot 8^{\mathrm{c}}$ \\
60 & - & $201 \cdot 2 \pm 7 \cdot 1$ & $4 \cdot 3 \pm 0 \cdot 5^{\mathrm{a}}$ & $3 \cdot 7 \pm 1 \cdot 4^{\mathrm{b}}$ & $92 \cdot 8 \pm 2 \cdot 5^{\mathrm{a}}$ \\
60 & + & $269 \cdot 0 \pm 9 \cdot 6$ & $2 \cdot 75 \pm 0 \cdot 5^{\mathrm{bc}}$ & $3 \cdot 2 \pm 1 \cdot 0^{\mathrm{b}}$ & $92 \cdot 0 \pm 2 \cdot 6^{\mathrm{a}}$ \\
40 & - & $138 \cdot 0 \pm 4 \cdot 9$ & $2 \cdot 0 \pm 0 \cdot 4^{\mathrm{c}}$ & $3 \cdot 4 \pm 1 \cdot 1^{\mathrm{b}}$ & $99 \cdot 1 \pm 0 \cdot 8^{\mathrm{a}}$ \\
40 & + & $201 \cdot 25 \pm 8 \cdot 1$ & $2 \cdot 0 \pm 0 \cdot 7^{\mathrm{c}}$ & $3 \cdot 5 \pm 0 \cdot 9^{\mathrm{b}}$ & $99 \cdot 2 \pm 0 \cdot 7^{\mathrm{a}}$ \\
20 & - & $71 \cdot 25 \pm 3 \cdot 0$ & $0 \cdot 25 \pm 0 \cdot 3^{\mathrm{d}}$ & $7 \cdot 2 \pm 3 \cdot 7^{\mathrm{b}}$ & $99 \cdot 9 \pm 0 \cdot 1^{\mathrm{a}}$ \\
20 & + & $122 \cdot 0 \pm 4 \cdot 7$ & $0 \cdot 50 \pm 0 \cdot 3^{\mathrm{d}}$ & $4 \cdot 9 \pm 1 \cdot 4^{\mathrm{b}}$ & $100 \cdot 0 \pm 0^{\mathrm{a}}$ \\
0 & - & $1 \cdot 0 \pm 0 \cdot 0$ & $0^{\mathrm{d}}$ & $59 \cdot 6 \pm 19 \cdot 3^{\mathrm{a}}$ & $100 \cdot 0 \pm 0^{\mathrm{a}}$ \\
0 & + & $47 \cdot 0 \pm 2 \cdot 5$ & $0^{\mathrm{d}}$ & $20 \cdot 1 \pm 16 \cdot 3^{\mathrm{b}}$ & $100 \cdot 0 \pm 0^{\mathrm{a}}$ \\
\hline
\end{tabular}

Values are mean \pm s.e.m. of 5 replicates.

Values with different superscript letters are significantly different $(P<0.05)$.

Although the 60, 40, 20,0\% BPSE dilutions with and without Ficoll resulted in damage to over $90 \%$ of the spermatozoa, progressive deterioration of the spermatozoa was detected with each increment in hypotonicity. Mitochondria first appeared swollen in $80 \%$ BPSE, and swelled more with each increment in hypotonicity (Pl. 2, Figs 9 and 11). In 0\% BPSE without Ficoll spermatozoa were nearly spherical due to coiling of the tail. With Ficoll, the coiled tails were seen to be undulating within the distended plasmalemma (Pl. 2, Figs 11 and 13). As with the fowl spermatozoa, the halo appeared to move along the length of the head and coiled tail of immotile cells. Swollen mitochondria that had become dislodged were observed free within the distended plasmalemma.

\section{Motility and percentage dead}

These values are given in Tables 1 and 2. The increased viscosity of the BPSE due to the addition of Ficoll resulted in an overall depression in fowl and turkey sperm motility. Although motile in the Ficoll containing solutions, the spermatozoa were sluggish. The addition of Ficoll to the $0 \%$ BPSE solution significantly reduced the numbers of dead spermatozoa compared with those in $0 \%$ BPSE alone, but all of the spermatozoa were still immotile.

Table 3. Percentage fertility obtained with fowl and turkey spermatozoa in hypotonic BPSE

\begin{tabular}{|c|c|c|c|c|c|c|c|c|}
\hline \multicolumn{3}{|c|}{ Fowl } & \multicolumn{6}{|c|}{ Turkey } \\
\hline $\begin{array}{c}\text { BPSE } \\
(\%)\end{array}$ & $\begin{array}{l}\text { No. } \\
\text { of hens }\end{array}$ & Fertility* & $\begin{array}{c}\text { BPSE } \\
(\%)\end{array}$ & $\begin{array}{l}\text { No. } \\
\text { of hens }\end{array}$ & Fertility* & 2nd week ${ }^{\dagger}$ & 3 rd week $\ddagger$ & 4th week $\S$ \\
\hline 100 & 8 & $92^{a}$ & 100 & 7 & $92^{\mathrm{a}}$ & $91^{a}$ & $91^{\mathrm{a}}$ & $87^{a}$ \\
\hline 80 & 8 & $86^{\mathrm{a}}$ & 80 & 8 & $93^{\mathrm{a}}$ & $96^{\mathrm{a}}$ & $86^{\mathrm{a}}$ & $76^{\mathrm{a}}$ \\
\hline 60 & 7 & $51^{\mathrm{b}}$ & 60 & 8 & $84^{\mathrm{a}}$ & $66^{\mathrm{b}}$ & $58^{\mathrm{b}}$ & $55^{b}$ \\
\hline 40 & 9 & $17^{\mathrm{c}}$ & 40 & 8 & $16^{\mathrm{b}}$ & $13^{c}$ & $6^{\mathrm{c}}$ & $0^{c}$ \\
\hline 20 & 7 & $11^{\mathrm{c}}$ & 20 & 8 & $8^{\mathrm{bc}}$ & $3^{\text {cd }}$ & $2^{\mathrm{c}}$ & $0^{c}$ \\
\hline 0 & 6 & $6^{c}$ & 0 & 8 & $0^{\mathfrak{c}}$ & $0^{d}$ & $2^{c}$ & $0^{c}$ \\
\hline
\end{tabular}

Values with a different superscript letter are significantly different $(P<0.05)$.

* Based on average \% fertility for each hen for a 4-week period.

+ Based on average \% fertility for each hen for Days 8-14 after last insemination.

$¥$ Based on average \% fertility for each hen for Days 15-21 after last insemination.

$\S$ Based on average \% fertility for each hen for Days $22-28$ after last insemination. 
Fertility

As shown in Table 3, the fertility of fowl was decreased when spermatozoa in $60 \%$ BPSE were used. For turkey spermatozoa, a significant depression of fertility was seen with $40 \%$ BPSE and no fertile eggs were obtained with 0\% BPSE.

There was little effect of hypotonic BPSE on prolonged fertility of turkeys: only in the $60 \%$ BPSE group did fertility decrease significantly during the 2nd week after AI (Table 3).

To assess the affect of Ficoll on fertility, 7 fowl hens were inseminated weekly with $100 \%$ BPSE plus $20 \%$ Ficoll. After 4 weeks fertility was $85 \%$, indicating that Ficoll had a slight depressing effect on fertility when compared to the value of $92 \%$ (Table 3 ) for $100 \%$ BPSE without Ficoll.

\section{Discussion}

Lake (1954) recognized that the head-midpiece junction of fowl spermatozoa is susceptible to environmentally induced damage (disruption of mitochondria and bending) and such damage must be prevented in order to ensure optimal fertility. Tsukunaga \& Takahashi (1961) and Yamane et al. (1966) suggested that the occurrence of bent spermatozoa is a manifestation of midpiece hydration and/or disruption that is limited to motile spermatozoa exposed to hypotonic conditions. The observations in this study support, in part, those of Lake (1954), Tsukunaga \& Takahashi (1961) and Yamane et al. (1966). We found that the disruption of the midpiece may be limited to the distension of the plasmalemma overlying the midpiece and does not necessarily affect the mitochondrial sheath. Mitochondrial swelling, however, is prevalent when spermatozoa are exposed to the more hypotonic solutions. Distension of the plasmalemma at the midpiece may predispose this segment to further damage such as bending and mitochondrial swelling. Plasmalemma distension at the midpiece appears to precede or is concomitant with formation of bent spermatozoa as a distended plasmalemma was always accompanied by distension of the plasmalemma in that region.

Dickey (1965) suggested that bent spermatozoa in the bovine epididymis were related to the translocation of the cytoplasmic droplet which completely encloses the bend. Bent bovine spermatozoa in ejaculates are motile, although they move with the droplet in a forward position and with the head trailing (Saacke, 1970).

Our observations on motility, percentage dead, and morphology gave no indication why the fertility of fowl spermatozoa in $60 \%$ BPSE should be less than that of turkey spermatozoa. The numbers of spermatozoa inseminated differed $\left(250 \times 10^{6} /\right.$ week in turkey and $150 \times 10^{6} /$ week in fowl) but the percentages of abnormal spermatozoa were similar, with almost all the motile spermatozoa being bent. Considering that the number of bent spermatozoa is inversely proportional to fertility (Saeki, 1960) and that about $90 \%$ of the spermatozoa were judged abnormal with bent spermatozoa being the predominant anomaly, it is surprising that fertility in the $60 \%$ BPSE treatment was as high as $84 \%$ in the turkeys. If all morphologically abnormal spermatozoa are considered infertile then the remaining $10 \%$ normal turkey spermatozoa were responsible for this high fertility value. It is, however, possible that all morphologically abnormal spermatozoa may not be infertile; some spermatozoa with anomalies induced by hypotonicity may assume normal morphology when exposed to isotonic conditions in the vagina. Fowl spermatozoa that are immotile in 0\% BPSE become motile when placed in an equal volume of $100 \%$ BPSE and although many spermatozoa remained bent, cells of normal appearance were observed. Immotile, swollen bovine spermatozoa with coiled tails in hypotonic media gradually assumed normal appearance and motility patterns as the medium was brought to near isotonicity (Drevius \& Ericksson, 1966).

Although several variations of bent spermatozoa have been described based on the location and degree of the tail flexure (Tsukunaga \& Takahashi, 1961: Yamane et al., 1966), we limited our description to those cells with an acute bend at the midpiece and to those with a looped bend 
around the proximal portion of the principal piece. The latter type appears to represent a more gradual response of the spermatozoon to increased intracellular water volume and thus is more likely to return to a normal morphology when exposed to isotonic conditions. Those spermatozoa with an acute bend at the midpiece may be permanently damaged. Supporting this suggestion is the observation that immotile bent spermatozoa lacking a halo in Ficoll-containing solution and presumed dead have an acute bend at the midpiece.

The type of damage observed in this study is nearly identical to that reported by Bakst \& Sexton (1979) resulting from pre- and post-freezing treatments of fowl and turkey spermatozoa. Plasmalemma distension, bent spermatozoa, coiled tails and mitochondrial swelling were observed by scanning electron microscopy after each step of the freezing process and were attributed to adverse osmotic conditions. Turkey spermatozoa appeared more susceptible to preand post-freezing treatments although qualitatively the damage to fowl and turkey spermatozoa was identical. In this study, turkey spermatozoa also appeared more susceptible to plasmalemma distension and mitochondrial swelling, and this may indicate differences in plasmalemma permeability or that the midpiece of the turkey spermatozoon is more hypertonic, with respect to its environment, than that of the fowl spermatozoon.

Using phase-contrast microscopy to examine spermatozoa in a hypotonic medium with a high refractive index permits assessment of the integrity of the sperm plasmalemma. With the assumption that spermatozoa with an intact plasmalemma (showing a halo) are alive, this procedure is being compared to nigrosin/eosin staining for possible application as a live/dead indicator of spermatozoa.

I thank Ms G. Kaminski for her expert assistance.

\section{References}

Bakst, M.R. \& Sexton, T.J. (1979) Fertilizing capacity and ultrastructure of fowl and turkey spermatozoa before and after freezing. J. Reprod. Fert. 55, 1-7.

Barr, J.A., Goodnight, J.H., Sell, J.P. \& Helwig, J.T. (1976) A Users Guide to SAS 76. Sparks Press, Raleigh, North Carolina.

Dickey, J.F. (1965) An electron microscope study of bovine spermatozoa undergoing maturation in the male reproductive system. Ph.D. thesis, Pennsylvania State University.

Drevius, L. (1972) Bull spermatozoa as osmometers. $J$. Reprod. Fert. 28, 29-39.

Drevius, L. \& Ericksson, H. (1966) Osmotic swelling of mammalian spermatozoa. Exp. Cell Res. 42, 136-156.

Harris, G.C., Thurston, R.J. \& Cundall, J. (1973) Changes in the ultrastructure of the fowl spermatozoa due to rapid freeze-thaw. J. Reprod. Fert. 34, 389-394.

Lake, P. (1954) The relationship between morphology and function in fowl spermatozoa. Proc. 10th Wld Poult. Congr., Edinburgh pp. 79-85.

Marquez, B. \& Ogasawara, F. (1977) Ultrastructural changes in turkey spermatozoa after immersion in glycerolyzed media and during various steps used in cryopreservation. Poultry Sci. 56, 1806-1813.

Oderkirk, A.H.F. \& Buckland, R.B. (1976) A comparison of diluents and cryopreservatives for freezing turkey semen. Poultry Sci. 55, 2073, Abstr.

Polge, C. (1951) Functional survival of fowl spermatozoa after freezing at $-70^{\circ} \mathrm{C}$. Nature, Lond. $167,949-950$.
Saacke, R.G. (1970) Morphology of the sperm and its relationship to fertility. Proc. 3rd Tech. Conf. Anim. Reprod. \& A.I., Nat. Ass. Anim. Breeders, Chicago, pp. 3-16.

Saeki, Y. (1960) Crooked-neck spermatozoa in relation to low fertility in the artificial insemination of fowl. Poultry Sci. 39, 1354-1361.

Sexton, T. (1976) Studies on the fertility of frozen fowl semen. Proc. 8th Int. Congr. Anim. Reprod. \& A.I., Krakow, pp. 1079-1081.

Sexton, T. (1977) A new poultry semen extender. I. Effect of extension on the fertility of chicken semen. Poultry Sci. 56, 1443-1446.

Tsukunaga, S. \& Takahashi, T. (1961) The promotion of fertility rate of cock semen by adjusting the osmotic pressure of yolk-citrate buffer. Bull. Hiroshima Agric. Coll. 1, 25-34.

Van Wambeke, F. (1977) The effect of tonicity of storage media for fowl semen on the occurrence of neck-bending spermatozoa, fertility and hatchability. Br. Poultry Sci. 18, 163-168.

Westfall, F. \& Harris, G. (1975) The ability of cryopreservatives to prevent motility loss and frozenthaw damage to the acrosome of chicken sperm. Cryobiology 12, 89-92.

Yamane, J., Tsukunaga, S. \& Takahashi, T. (1966) Hiroshima method of artificial insemination of the domestic fowl. J. Fac. Fish. Anim. Husb. Hiroshima Univ. 6, 395-429. 